\title{
Fluxonium-Based Artificial Molecule with a Tunable Magnetic Moment
}

\begin{abstract}
A. Kou, 'W. C. Smith, U. Vool, R. T. Brierley, H. Meier, L. Frunzio, S. M. Girvin, L. I. Glazman, and M. H. Devoret
Departments of Applied Physics and Physics, Yale University, New Haven, Connecticut 06520, USA

(Received 5 October 2016; revised manuscript received 28 February 2017; published 29 August 2017)

Engineered quantum systems allow us to observe phenomena that are not easily accessible naturally. The LEGO®-like nature of superconducting circuits makes them particularly suited for building and coupling artificial atoms. Here, we introduce an artificial molecule, composed of two strongly coupled fluxonium atoms, which possesses a tunable magnetic moment. Using an applied external flux, one can tune the molecule between two regimes: one in which the ground-excited state manifold has a magnetic dipole moment and one in which the ground-excited state manifold has only a magnetic quadrupole moment. By varying the applied external flux, we find the coherence of the molecule to be limited by local flux noise. The ability to engineer and control artificial molecules paves the way for building more complex circuits for quantum simulation and protected qubits.
\end{abstract}

DOI: 10.1103/PhysRevX.7.031037

Subject Areas: Mesoscopics, Quantum Information, Superconductivity

\section{INTRODUCTION}

Superconducting circuits are a flexible platform for building artificial atoms. By choosing the proper combination of inductors, capacitors, and Josephson junctions, the experimentalist can tailor superconducting circuits to be governed by a variety of Hamiltonians [1-6]. A particular example is the fluxonium atom, which consists of a small Josephson junction in parallel with a superinductance $[7,8]$. Its spectrum maintains the anharmonic structure associated with single Cooper-pair phenomena while suppressing the detrimental effect of offset charges. At the external flux sweet spot, its ground-excited state manifold, $\{|g\rangle,|e\rangle\}$, is rendered flux-noise insensitive to first order.

Given the success of building artificial atoms using superconducting circuits, one may wonder whether we can build novel artificial molecules. Previous work on coupled superconducting circuits [9-13] has focused on the regime where the ground-state configuration of the coupled system remains essentially localized within individual artificial atoms. Artificial molecules, on the other hand, would have degrees of freedom that are delocalized between constituent artificial atoms, in analogy with the electronic motion that is delocalized between several nuclei in natural molecules. Here, the experimentalist would be able to tailor the type and strength of coupling between individual artificial atoms. In particular, by cleverly choosing the coupling between constituent atoms, the experimentalist could make the states of an artificial

\footnotetext{
* Corresponding author. angela.kou@yale.edu

Published by the American Physical Society under the terms of the Creative Commons Attribution 3.0 License. Further distribution of this work must maintain attribution to the author(s) and the published article's title, journal citation, and DOI.
}

molecule sensitive to different components of external fields. As an example, we can consider molecules with magnetic dipole moments versus molecules with only magnetic quadrupole moments. Magnetic dipoles are sensitive to uniform magnetic fields, while magnetic quadrupoles are only sensitive to magnetic-field gradients. In natural molecules, however, the order of the magnetic moment cannot easily be changed.

The novelty of a tunable synthetic molecule extends to superconducting-circuit-based quantum simulation, where such a device presents a crucial intermediate step. Delocalized ground states are a hallmark of strongly correlated many-body systems, and an artificial molecule is the smallest system that can demonstrate such states. Quantum simulators must faithfully obey a given many-body Hamiltonian and should be capable of accessing multiple phases of the physical system under investigation. Before building an interacting large- $N$ system, where $N$ is the number of artificial atoms, such as the one proposed in Ref. [14], however, one must first experimentally realize and control an artificial molecule composed of $N=2$ interacting artificial atoms.

Here, we report an experiment in which we build an artificial molecule whose magnetic moment can be tuned via an applied external flux. The artificial molecule is composed of two fluxonium atoms strongly coupled via a shared inductance. At low applied external flux, the ground state $|g\rangle$ is a separable state comprising persistent currents flowing in the same direction, while the excited state $|e\rangle$ is an odd superposition of persistent currents flowing in opposite directions. The $\{|g\rangle,|e\rangle\}$ manifold is predominantly sensitive to common-mode flux noise that affects both atoms simultaneously. As the applied external flux is increased, $|g\rangle$ changes character and becomes the even superposition of persistent currents flowing in opposite 
directions, while $|e\rangle$ essentially retains its symmetry. An energy gap that is about 10 times larger than the groundexcited state transition energy in a single fluxonium atom at its external flux sweet spot, determined by the substantial shared superinductance, decouples external-flux-dependent states from the external-flux-independent states in the $\{|g\rangle,|e\rangle\}$ manifold. While this $\{|g\rangle,|e\rangle\}$ manifold has become insensitive to common-mode flux noise, it remains sensitive to differential-mode flux noise. Previous experiments have addressed the common-mode and differentialmode flux-noise dichotomy but only near the flux-noise sweet spot $[12,15,16]$. Here, we perform spectroscopy on the fluxonium-based molecule and observe transitions between the ground state and excited states over multiple quanta of applied external flux. We investigate decoherence mechanisms in the device and conclude that the coherence of the $|g\rangle-|e\rangle$ transition is limited by local flux noise.

\section{THEORETICAL MODEL}

Our system is composed of two fluxonium atoms coupled via a shared inductance as shown in Fig. 1(a). The artificial molecule obeys the Hamiltonian:

$$
\begin{aligned}
H= & 4 E_{C}\left(n_{1}^{2}+n_{2}^{2}\right)+\frac{1}{3} E_{L}\left(\varphi_{1}^{2}+\varphi_{2}^{2}+\varphi_{1} \varphi_{2}\right) \\
& -E_{J} \cos \left(\varphi_{1}-\frac{2 \pi}{\Phi_{0}} \Phi_{1}\right)-E_{J} \cos \left(\varphi_{2}-\frac{2 \pi}{\Phi_{0}} \Phi_{2}\right),
\end{aligned}
$$

where $E_{C}$ is the charging energy of each junction; $n_{1}$ and $n_{2}$ are the number of Cooper pairs on each junction capacitor plate; $E_{L}$ is the inductive energy associated with each superinductance; $E_{J}, \varphi_{1}, \varphi_{2}$ are, respectively, the Josephson energies and the phase differences across each of the junctions; $\Phi_{1}$ and $\Phi_{2}$ are the fluxes in each loop; and $\Phi_{0}$ is the magnetic flux quantum. Here, we have assumed that the small junctions are identical. The $\varphi_{1} \varphi_{2}$ term reflects the inductive coupling in the circuit and corresponds to its "molecular" aspect. This coupling term has a positive sign and favors opposite phase differences across the two Josephson junctions. The two fluxonium atoms are strongly coupled; the strength of the coupling term in the artificial molecule is equal to the inductive energy of each individual fluxonium atom $\left(E_{L} / h \sim 1 \mathrm{GHz}\right)$.

With the help of a gauge transformation [i.e., a shift of the phases $\varphi_{i}$ to $\left.\varphi_{i}+\left(2 \pi / \Phi_{0}\right) \Phi_{i}, i=1,2\right]$, we can rewrite Eq. (1) as

$$
\begin{aligned}
H= & 4 E_{C}\left(n_{1}^{2}+n_{2}^{2}\right) \\
& +\frac{1}{4} E_{L}\left[\left(\varphi_{1}+\varphi_{2}+\varphi_{\mathrm{com}}\right)^{2}+\frac{1}{3}\left(\varphi_{1}-\varphi_{2}+\varphi_{\text {diff }}\right)^{2}\right] \\
& -2 E_{J} \cos \left(\frac{\varphi_{1}+\varphi_{2}}{2}\right) \cos \left(\frac{\varphi_{1}-\varphi_{2}}{2}\right)
\end{aligned}
$$

where we have introduced the common and differential applied reduced fluxes, $\varphi_{\text {com }}=\left[(2 \pi) / \Phi_{0}\right]\left(\Phi_{1}+\Phi_{2}\right)$ and $\varphi_{\text {diff }}=\left[(2 \pi) / \Phi_{0}\right]\left(\Phi_{1}-\Phi_{2}\right)$. This form of the Hamiltonian illustrates the sensitivity of the molecule to both modes of magnetic-field fluctuations. If the spatial field variation is small on the scale of the device, then $\varphi_{\text {com }}$ and $\varphi_{\text {diff }}$ are proportional to the value of the magnetic field and its gradient at the position of the device, respectively. Therefore, we define the artificial molecule to be "dipolar" when it is sensitive to $\varphi_{\text {com }}$ and $\varphi_{\text {diff }}$ and "quadrupolar" when it is only sensitive to the differential flux $\varphi_{\text {diff }}$.

The artificial molecule is operated in the two regimes by changing an applied external magnetic flux, $\Phi_{\text {ext }}$, which sets the temporal average of the common-mode flux $\overline{\left(\Phi_{1}+\Phi_{2}\right)} / 2=\Phi_{\text {ext }}$, and, to a much weaker extent, the temporal average of the differential-mode flux $\overline{\left(\Phi_{1}-\Phi_{2}\right)}=\alpha \Phi_{\text {ext }}$, where $\alpha \ll 1$. In the first regime, $0 \leq \Phi_{\text {ext }} \lesssim 0.3 \Phi_{0}$, the molecule essentially behaves like a magnetic dipole. The phase difference across both of the junctions in the molecule is the same. As shown in Figs. 1(b) and 1(c), the potential has one deep well centered near $\varphi_{1}=0$ and $\varphi_{2}=0$, and the ground state is localized in this well. This ground state is simply the product of the single fluxonium-atom ground states and corresponds to currents flowing in the same direction in both fluxonium atoms. The direction of current flow is determined by the direction of the applied external flux. It helps to think of the persistent current chirality as the spin of the fluxonium atom. The excited state, then, corresponds to the singlet spin state, while the ground state corresponds to the $m=1$ triplet spin state. To transition from $|g\rangle$ to $|e\rangle$, the persistent current in one of the fluxonium atoms needs to change direction, which is analogous to flipping a spin. The transition frequency here is then determined by the applied external flux and is, hence, sensitive to noise in the common-mode flux.

As the external flux is increased, the two fluxonium atoms start to behave like a molecule with no dipole moment but with a quadrupole moment. In this regime, the potential landscape of the molecule has two degenerate potential wells as shown in Fig. 1(e). The degeneracy of the wells comes from the symmetry between $\varphi_{1}$ and $\varphi_{2}$ in the Hamiltonian [Eq. (1)]. These two wells correspond to the two possible configurations of persistent currents flowing in opposite directions in each loop. The energy splitting between $|g\rangle$ and $|e\rangle$ is determined by the tunneling between the two lowest degenerate potential wells.

The ground-excited state manifold of the molecule near $\Phi_{\text {ext }}=0.5 \Phi_{0}$ is formed from superpositions of the currents flowing in opposite directions in the two fluxonium atoms as shown in Figs. 1(d) and 1(e). The $|g\rangle$ state thus corresponds to the $m=0$ triplet spin state, and the $|e\rangle$ state corresponds to the singlet spin state. This groundexcited state manifold is gradiometric; the transition is insensitive to fluctuations in external flux that are uniform across both loops. We note that in the limit of perfect 
(a)
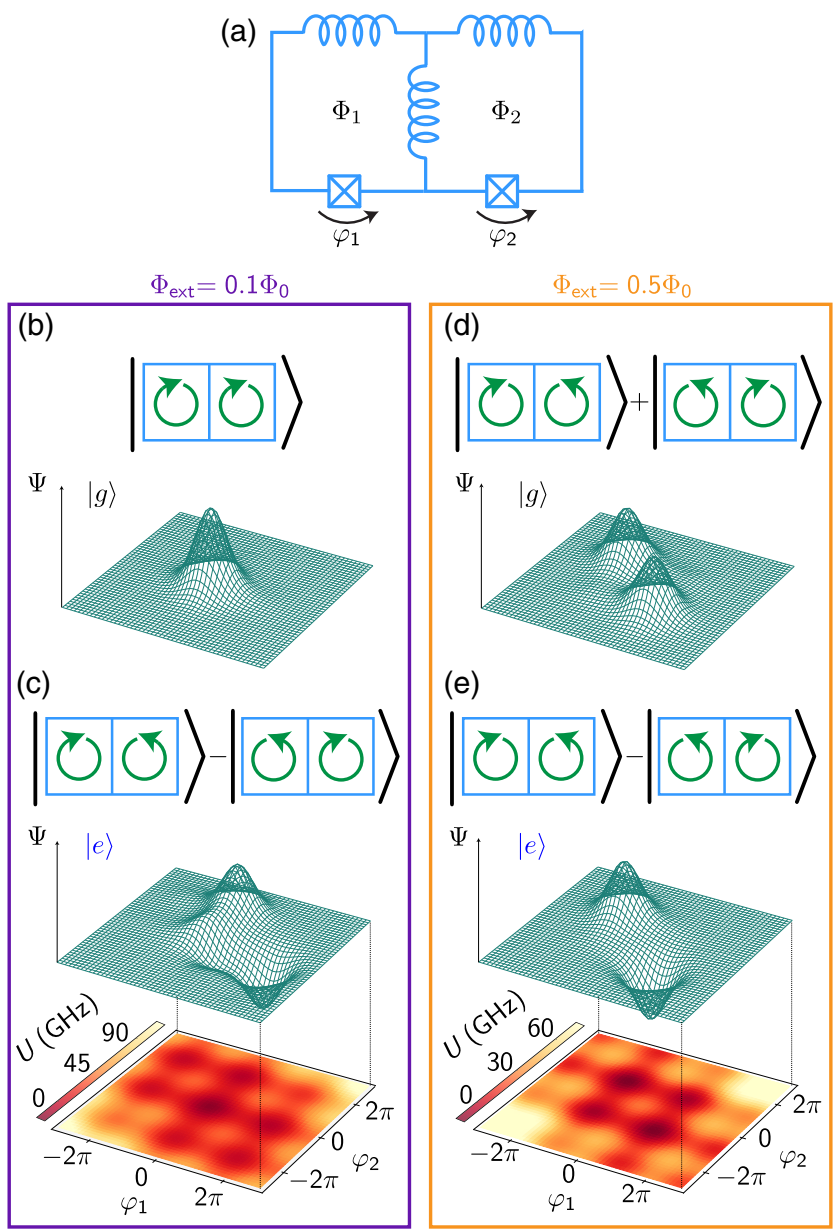

(e)
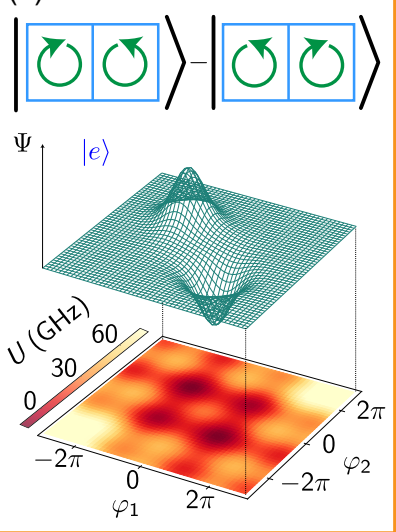

FIG. 1. (a) Electrical circuit diagram of the artificial molecule. (b,c) Magnetic dipole regime: Ground- and excitedstate wave functions $(\Psi)$, and potential $(U)$ at $\Phi_{\text {ext }}=$ $\overline{\left(\Phi_{1}+\Phi_{2}\right)} / 2=0.1 \Phi_{0}$. The direction of persistent current flow in the ground state is indicated with green arrows. The ground state is a product state of the two fluxonium atoms and is localized in the potential well near $\varphi_{1}, \varphi_{2}=0$. The excited state corresponds to persistent currents flowing in opposite directions and is delocalized in multiple potential wells. (d,e) Magnetic quadrupole regime: Approximate kets for the ground and excited states at $\Phi_{\text {ext }}=0.5 \Phi_{0}$. Ground-state and excited-state wave functions $(\Psi)$ are shown above the potential $(U)$ of the artificial molecule. The ground and excited states are symmetric and antisymmetric superpositions of persistent currents flowing in opposite directions. The wave functions are localized in the two lowest potential wells, which are degenerate. Higher-energy excited states tend to be localized in the shallower potential wells.

symmetry between the two fluxonium atoms, this groundexcited state manifold is also insensitive to first order to fluctuations in external flux that occur only in individual loops.

As a consequence of this insensitivity, $f_{g e}$ is nearly constant over a large range in $\Phi_{\text {ext }}$, which should protect this transition from common-mode flux noise. Another way to see this is to consider that both junctions must undergo a quantum phase slip in order to tunnel between the two lowest potential wells. The persistent currents in both fluxonium atoms must flip direction together. A double phase slip is a second-order process where the molecule has to make a virtual transition through a higher-energy state with the currents circulating in the same direction in the two fluxonium loops. The energy splitting is then on the order of $E_{S}^{2} / \Delta$, where $E_{S} \sim\left(E_{J}^{3} E_{C}\right)^{1 / 4} e^{-\sqrt{8 E_{J} / E_{C}}}$ is the energy of a single phase slip in an individual fluxonium atom [14,17] and $\Delta \sim \frac{2}{3} \pi^{2} E_{L}$ is the energy difference between the states with counter-circulating currents in the two loops and the states with currents flowing in the same direction in the two loops. We note here that the decoherence rate associated with an unwanted phase slip will be suppressed by a factor of $E_{S} / \Delta$ when compared with the single fluxonium atom.

\section{EXPERIMENTAL REALIZATION}

The artificial molecule device (device A) is shown in Fig. 2(a). Each fluxonium atom consists of a small Josephson junction, which provides nonlinearity, in series with an array of 40 larger junctions. The array of 40 junctions has an inductance of $140 \mathrm{nH}$ and serves as the superinductance for each atom [18]. The fluxonium atoms are connected in parallel with an additional array of 40 junctions, which provide inductive coupling between the atoms. The artificial molecule is inductively coupled to a readout antenna via shared Josephson junctions, which have an inductance of $3.0 \mathrm{nH}$.

The inductively loaded readout antenna is a LC oscillator, where the inductance is provided by 14 Josephson junctions and the capacitance is provided by the long metal electrodes as shown in Fig. 2(b). The antenna has a

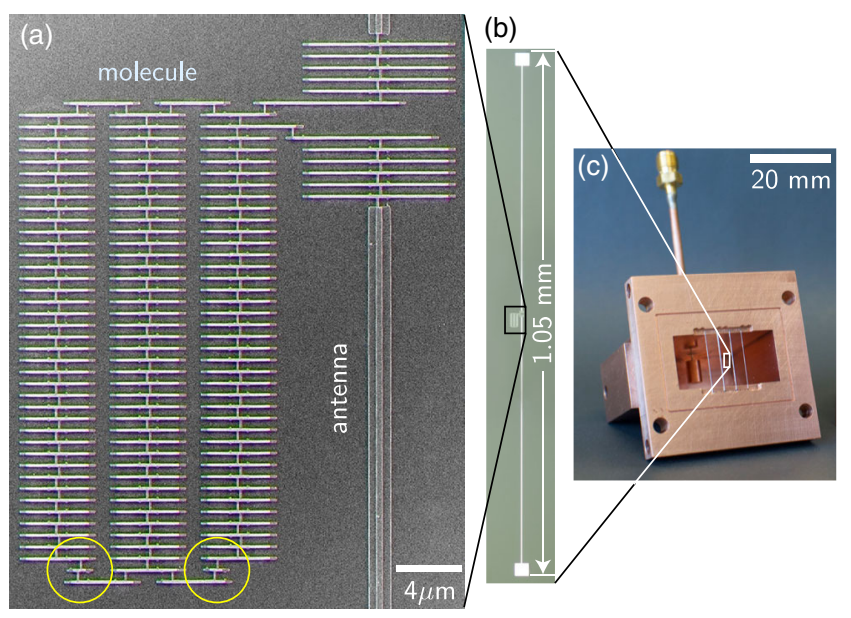

FIG. 2. (a) Scanning electron micrograph of device A. Small junctions are indicated in the yellow circles. The artificial molecule is coupled via Josephson junctions to the readout antenna whose optical image is shown in (b). (c) The molecule and antenna are fabricated on a sapphire chip, which is then placed inside a copper waveguide. 
resonant frequency of $f_{a}=7.875 \mathrm{GHz}$ and a FWHM linewidth of $\kappa / 2 \pi=6 \mathrm{MHz}$. The junctions were fabricated with $\mathrm{Al} / \mathrm{AlO}_{x} / \mathrm{Al}$ using the bridge-free double-angle evaporation technique [19]. Both the molecule and the antenna were fabricated on a sapphire chip. This chip was then placed inside an impedance-matched copper waveguide [Fig. 2(c)], which couples propagating microwaves to the molecule-antenna system. The waveguide was thermally anchored to the mixing chamber stage of a dilution refrigerator with a base temperature of about $16 \mathrm{mK}$. The waveguide was magnetically shielded by an aluminum shield coated with infrared-absorbing material, which was itself housed inside a cryogenic $\mu$-metal shield [20].

\section{MOLECULAR SPECTROSCOPY}

We used standard dispersive readout [21] to measure the molecule with the readout antenna. We performed a twotone spectroscopy experiment at different flux points to obtain the data shown in open circles in Fig. 3. We first applied a fixed $\Phi_{\text {ext }}$ through the device and then shone a microwave tone to excite the molecule at its resonant frequency, which resulted in a change in the resonant frequency of the readout antenna. The sample was biased via a large magnetic-field coil that encircles the entire device. This experiment was then repeated at multiple flux points between $\Phi_{\text {ext }} \sim-0.1 \Phi_{0}$ and $\Phi_{\text {ext }} \sim 1.5 \Phi_{0}$. We observed transitions between $|g\rangle$ and the first three excited states.

At the critical point (indicated by the start of the grey shaded area in Fig. 3) at $\Phi_{\text {ext }} \approx 0.3 \Phi_{0}$, we observed that the transition frequency from $|g\rangle$ to $|f\rangle$ starts to increase as a function of flux. For $\Phi_{\text {ext }}>0.3 \Phi_{0}$, the $|g\rangle-|e\rangle$ transition frequency $f_{g e}$ becomes very small. At its lowest point, $f_{g e}$ reaches $105 \mathrm{MHz}$ at $\Phi_{\text {ext }}=0.5 \Phi_{0}$. In contrast with other flux-based qubits such as the flux qubit [2] and the fluxonium [7], $f_{g e}$ is also remarkably flat as a function of $\Phi_{\text {ext }}$ near $\Phi_{\text {ext }}=0.5 \Phi_{0}$, as shown in Fig. 3 .

At $\Phi_{\text {ext }}=0.3 \Phi_{0}$, the potential landscape of the system transitions from having a single lowest potential well to having two degenerate potential wells. The former ground state of the molecule-currents circulating in the same direction in both loops-becomes the $|f\rangle$ state. For $\Phi_{\text {ext }}>0.3 \Phi_{0}$, the $|g\rangle$ and $|e\rangle$ states are superpositions of currents flowing in opposite directions in the two loops as shown in Figs. 1(d) and 1(e). The energy splitting between $|g\rangle$ and $|e\rangle$ in this regime is $\sim E_{S}^{2} / \Delta$, which results in the very small $f_{g e}$.

We also note that the artificial molecule has different $|g\rangle-|e\rangle$ transition frequencies at external flux points
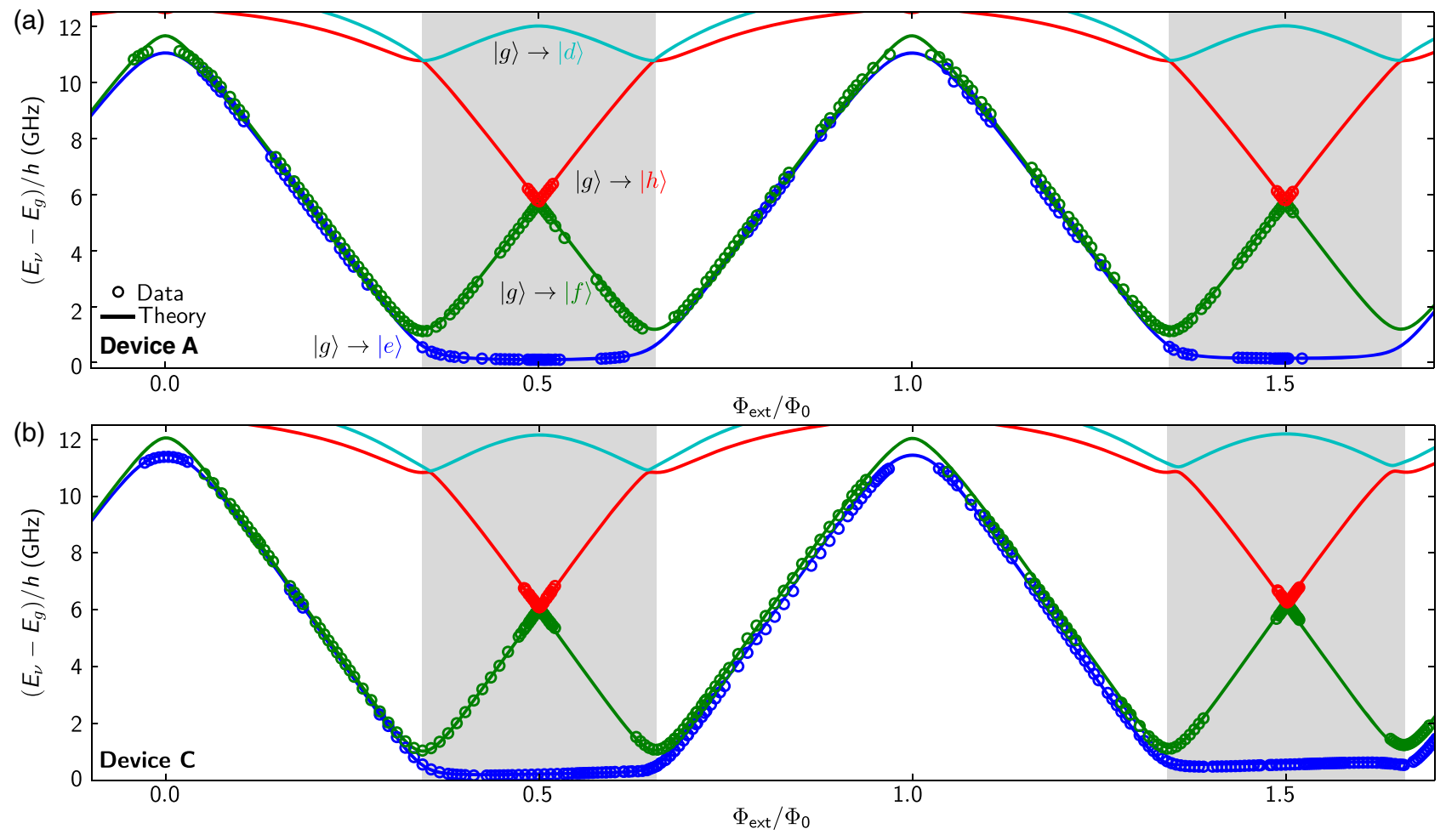

FIG. 3. (a,b) Transition frequency from $|g\rangle$ to excited states $|e\rangle,|f\rangle,|h\rangle,|d\rangle$ in device A and device C. Measured data are indicated with open circles. The solid lines were fit to the data using the Hamiltonian in Eq. (1) and the definition of $\Phi_{\text {ext }}$ (see text). Grey shaded areas indicate where the $|g\rangle-|e\rangle$ transition is only quadrupolar. Device $\mathrm{C}$ has a higher asymmetry between the two loops, which leads to a larger difference between the $|g\rangle$ to $|e\rangle$ transition frequency at $\Phi_{\text {ext }}=0.5 \Phi_{0}$ and the $|g\rangle$ to $|e\rangle$ transition frequency at $\Phi_{\text {ext }}=1.5 \Phi_{0}$ when compared with device A. 
separated by a flux quantum, which is to be expected in a device with unequal fluxonium loop areas. The $|g\rangle-|e\rangle$ transition frequency at $\Phi_{\text {ext }}=1.5 \Phi_{0}$ is $40 \mathrm{MHz}$ higher than $f_{g e}$ at $\Phi_{\text {ext }}=0.5 \Phi_{0}$ in device A.

Differences in the flux through the two fluxonium loops result in $\overline{\Phi_{1}-\Phi_{2}}=\alpha \Phi_{\text {ext }} \neq 0$ in the Hamiltonian [Eq. (1)]. The two fluxonium atoms then have different potential energies at each flux point. As the applied external flux is increased, this difference in potential landscapes compounds. The potential wells corresponding to currents flowing in opposite directions in the two loops are then no longer close to degenerate, resulting in the observed larger energy splitting at $\Phi_{\text {ext }}=1.5 \Phi_{0}$.

We compare the measured resonant frequencies (open circles in Fig. 3) with the theoretical prediction for these transition frequencies (solid lines in Fig. 3) obtained from numerical diagonalization of the Hamiltonian [Eq. (1)] [22]. The only asymmetry we have considered here is the nonzero $\alpha$ parameter. To fit the data, we constrain the product $E_{J} E_{C}$ based on fabrication parameters and effectively fit the full spectrum using only three fit parameters, $\left\{\alpha, E_{J} / E_{C}, E_{L}\right\}$. We find excellent agreement between the model and the data over three decades in transition frequencies (from $11.2 \mathrm{GHz}$ at $\Phi_{\text {ext }}=0$ to $105 \mathrm{MHz}$ at $\left.\Phi_{\text {ext }}=0.5 \Phi_{0}\right)$. This validates the approach of planning the behavior of a complex circuit, such as the one shown in Fig. 2, from a reduced set of engineerable parameters.

We further tested the validity of our approach by measuring multiple devices. The fit parameters for the measured devices are shown in Table I. The spectroscopy of device $\mathrm{C}$, which intentionally had the largest asymmetry between the two fluxonium loops, is shown in Fig. 3(b). For this device, the fit parameter $\alpha$ is consistent with the asymmetry inferred from optical images. We note again that the agreement between the theoretical fit and the measured data here is excellent. The effects of asymmetry are much more pronounced in this device. The lowest $f_{g e}=$ $197 \mathrm{MHz}$ no longer occurs at $\Phi_{\text {ext }}=0.5 \Phi_{0}$ but rather at $\Phi_{\text {ext }}=0.43 \Phi_{0}$. In addition, $f_{g e}$ at $\Phi_{\text {ext }}=1.5 \Phi_{0}$ is now $354 \mathrm{MHz}$ greater than $f_{g e}$ at $\Phi_{\mathrm{ext}}=0.5 \Phi_{0}$.

\section{TIME DOMAIN MEASUREMENTS}

In order to demonstrate the insensitivity of the molecule to certain decoherence mechanisms, we measured the coherence times of the $|g\rangle-|e\rangle$ transition for the three devices (A, B, and C) near $\Phi_{\text {ext }}=0.5 \Phi_{0}$. We performed standard time-domain measurements of the relaxation time $\left(T_{1}\right)$, Ramsey dephasing time $\left(T_{2 \mathrm{R}}\right)$, and spin-echo dephasing time $\left(T_{2 \mathrm{E}}\right)$. The coherence times at $\Phi_{\text {ext }}=0.5 \Phi_{0}$ are shown in Table I. Relaxation times for the measured devices are between 50 and $300 \mu$ s. Ramsey coherence times for the measured devices are between 0.4 and $1.25 \mu \mathrm{s}$. Spin-echo experiments, where a $\pi$ pulse is inserted into the standard Ramsey sequence, increased the coherence times by a factor of 4 , indicating the presence of a low-frequency decoherence mechanism.

In order to understand the mechanisms for decoherence in this molecule, which has surprisingly low $T_{2}$ 's given $T_{1}$, we measured the Ramsey dephasing rate $\Gamma_{\phi, R}$ as a function of $\Phi_{\text {ext }}$. The dependence of the $f_{g e}$ transition on $\Phi_{\text {ext }}$ allows us to isolate the contributions of common-mode and differentialmode flux noise to dephasing of the molecule as shown in Fig. 4 (see Ref. [23] for details). Near $\Phi_{\text {ext }}=0.5 \Phi_{0}$, the coherence of the $|g\rangle-|e\rangle$ transition is limited by differentialmode flux noise, while at $\Phi_{\text {ext }} \lesssim 0.3 \Phi_{0}$, it is predominantly limited by common-mode flux noise.

The spectral density of noise resulting from an arbitrary source $\lambda$ is

$$
S_{\delta \lambda}(\omega)=\frac{1}{2 \pi} \int_{-\infty}^{\infty} d \tau\langle\delta \lambda(t) \delta \lambda(t+\tau)\rangle e^{-i \omega \tau},
$$

where $\omega$ is the frequency at which the spectral density is taken. For common-mode flux noise, $\lambda=$ $\left[\Phi_{0} /(2 \pi \sqrt{2})\right] \varphi_{\text {com }}$, and for differential-mode flux noise, $\lambda=\left[\Phi_{0} /(2 \pi \sqrt{2})\right] \varphi_{\text {diff }}$.

Flux noise is typically assumed to have a $1 / f$ spectrum, i.e., $S=A^{2} /|\omega|$, where $A$ is the flux-noise amplitude. The common-mode and differential-mode flux-noise amplitudes are given in Table II. We find upper bounds

TABLE I. Parameters of three different artificial molecule devices. Here, $E_{J}$ is the Josephson energy of each fluxonium atom, $E_{C}$ is the charging energy of each fluxonium atom, $E_{L}$ is the inductive energy associated with each superinductance, $\alpha$ is the asymmetry between the fluxes of each fluxonium atom, $f_{g e}$ is the molecule transition frequency from $|g\rangle$ to $|e\rangle$ at $\Phi_{\text {ext }}=0.5 \Phi_{0}, T_{1}$ is the relaxation time from $|e\rangle$ to $|g\rangle, T_{2 R}$ is the coherence time measured by a Ramsey experiment, and $T_{2 E}$ is the coherence time measured by a spin-echo experiment. All timedomain measurements are done at $\Phi_{\text {ext }}=0.5 \Phi_{0}$.

\begin{tabular}{lcccccccc}
\hline \hline & & & & \multicolumn{4}{c}{$\Phi_{\text {ext }}=0.5 \Phi_{0}$} \\
\cline { 5 - 9 } Device & $E_{J}(\mathrm{GHz})$ & $E_{C}(\mathrm{GHz})$ & $E_{L}(\mathrm{GHz})$ & $\alpha$ & $f_{g e}(\mathrm{MHz})$ & $T_{1}(\mu \mathrm{s})$ & $T_{2 R}(\mu \mathrm{s})$ & $T_{2 E}(\mu \mathrm{s})$ \\
\hline $\mathrm{A}$ & 9.4 & 3.4 & 1.2 & 0.006 & 105 & 60 & 0.45 & 1.6 \\
$\mathrm{~B}$ & 9.5 & 3.4 & 1.1 & 0.007 & 110 & 50 & 1.25 & 5.4 \\
$\mathrm{C}$ & 9.8 & 3.3 & 1.2 & 0.03 & 197 & 300 & 0.31 & 1.2 \\
\hline \hline
\end{tabular}



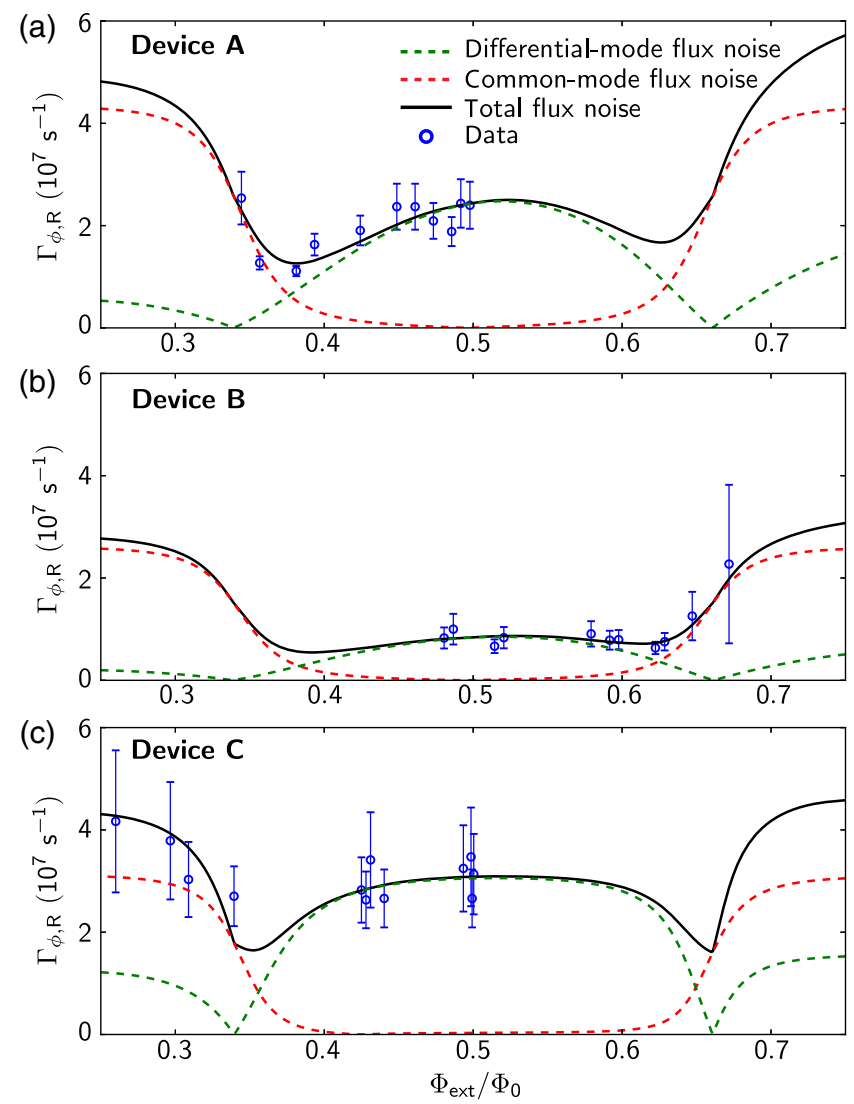

FIG. 4. (a)-(c) Ramsey dephasing rates as a function of flux in devices A, B, and C. The data are shown in open blue circles. Fit curves are the calculated Ramsey dephasing rates resulting from power spectral density values of differential-mode flux noise (green dashed), common-mode flux noise (red dashed), and their sum (black solid). We note that slight asymmetries in the two fluxonium loops cause the curves to be asymmetric around $\Phi_{\text {ext }}=0.5 \Phi_{0}$. This asymmetry also affects the amplitudes necessary to drive transitions and the visibility of the transitions between $|g\rangle$ and $|e\rangle$, resulting in the asymmetry in data of about $\Phi_{\text {ext }}=0.5 \Phi_{0}$ in these plots. Ramsey dephasing rate measurements could not be taken for the full range in $\Phi_{\text {ext }}$ for every sample because of the changing coupling between the molecule and readout antenna.

for flux-noise amplitudes between 4 and $11 \mu \Phi_{0}$. The fluxnoise amplitudes that we measure are somewhat larger but of the same order of magnitude as previous measurements of flux noise in flux qubits $[15,24,25]$ and coupled flux qubits [12].

Global flux noise, from a fluctuating applied magnetic field for example, would induce exclusively common-mode flux noise in the sample $\left(A_{\text {diff }} / A_{\text {com }}=0\right)$. On the other hand, local flux noise, such as that caused by spins fluctuating at the surface of the superconductor [26] or in defects at the metal-insulator interface [27], induces both common-mode and differential-mode flux noise in the sample. The exact branching ratio depends on the details of the model, but local flux noise will cause both commonmode and differential-mode flux noise on the unshared
TABLE II. Inferred flux-noise amplitudes from $T_{2 R}$ measurements together with the expected ratio of differential-mode to common-mode flux-noise amplitudes for each type of flux noise.

\begin{tabular}{lcccc}
\hline \hline & & \multicolumn{2}{c}{ Expected $A_{\text {diff }} / A_{\text {com }}$} \\
\cline { 3 - 5 } Device & $A_{\text {com }}\left(\mu \Phi_{0}\right)$ & $A_{\text {diff }}\left(\mu \Phi_{0}\right)$ & Global & Local \\
\hline A & $6 \pm 1$ & $10 \pm 2$ & 0 & $>1$ \\
B & $4 \pm 1$ & $4 \pm 1$ & 0 & $>1$ \\
$\mathrm{C}$ & $8 \pm 1$ & $11 \pm 2$ & 0 & $>1$ \\
\hline \hline
\end{tabular}

superinductances and differential-mode flux noise on the shared superinductance, resulting in a larger differential-mode contribution than common-mode contribution. Hence, for local flux noise, we would expect $A_{\text {diff }} / A_{\text {com }}>1$. We observe the latter behavior in all three devices. Near $\Phi_{\text {ext }}=0.5 \Phi_{0}$, the sample is insensitive to common-mode flux noise and is limited by differentialmode flux noise, which can only result from local sources.

We note that the residual asymmetry between the two loops in the molecule results in sensitivity to differentialmode flux noise (see Ref. [23]). A locally applied external flux could be used to compensate this asymmetry, which may result in longer coherence times. In addition, surface passivation has recently been found to decrease flux noise in superconducting quantum interference devices [28] and may also increase coherence times.

\section{CONCLUSION AND PERSPECTIVES}

In conclusion, we have realized an artificial molecule based on the fluxonium atom, which possesses a tunable magnetic moment. This molecule can be tuned from a weakly coupled regime where the ground state is a product state of the individual artificial atoms to a strongly coupled regime where the ground state is an entangled state with respect to the individual artificial atoms. A very basic circuit model accounts for all of the details observed in the spectroscopy of the molecule. This demonstrates that a complex superconducting circuit can be well-controlled, and its behavior can be predicted with few-parameter Hamiltonians. We have therefore taken a necessary step both toward creating artificial materials not realized in nature and toward quantum simulation with superconducting circuits where the Hamiltonian of the simulating system must faithfully map to the Hamiltonian of the system being simulated. We have also been able to perform spectroscopy of the molecule over multiple flux quanta and observe changes in its ground state from a separable state to an entangled state in situ. Longer chains of fluxonium atoms based on our artificial molecule have been predicted to show signatures of quantum phase transitions as the applied external flux is varied [14].

The $|g\rangle-|e\rangle$ transition of this molecule near $\Phi_{\text {ext }}=$ $0.5 \Phi_{0}$ is robust to excursions in common-mode flux, whereas for $\Phi_{\text {ext }} \lesssim 0.3 \Phi_{0}$, the transition is sensitive to 
excursions in both common-mode and differential-mode flux. The demonstrated insensitivity to common-mode flux noise is an example of symmetry-based protection. This type of protection relies on coupling strengths to a shared environment that are the same for the constituents of the molecule; i.e., it will protect the system from correlated noise sources. Such symmetry-based protection can supplement traditional quantum error correction, which corrects only independent qubit errors.

Surprisingly, the coherence of the ground-excited state manifold near $\Phi_{\text {ext }}=0.5 \Phi_{0}$ is not improved by this insensitivity to common-mode flux noise. Detailed measurements of $\Gamma_{\phi, R}$ as a function of $\Phi_{\text {ext }}$ pinpoint local flux noise as the main limitation for the coherence of these devices. Our work thus constitutes new evidence for the hypothesized local flux noise in superconducting circuits. Possible circuit-based solutions to this local source of decoherence are practical realizations of the proposed circuits of Ioffe et al. [29] and Brooks et al. [30]. Recent experiments performed on such circuits, formed from two coupled qubits composed of small Josephson junctions in parallel with a few large Josephson junctions, have been shown to protect against energy relaxation caused by charge noise [16,31]. The coherence of these circuits, however, remained limited by local flux noise differentially coupling to the slightly asymmetric qubits. Alternative fluxonium-based realizations of such circuits with a single loop may perform better in combating local flux noise [32].

\section{ACKNOWLEDGMENTS}

We acknowledge fruitful discussions with Rob Schoelkopf, Ioan Pop, Shyam Shankar, and Chen Wang, as well as support from the Yale Quantum Institute. Facilities use was supported by the Yale SEAS cleanroom, Yale Institute for Nanoscience and Quantum Engineering (YINQE), and NSF MRSEC Grant No. DMR-1119826. This research was supported by the Army Research Office under Grant No. W911NF-14-1-0011 and by Office for Naval Research under Grant No. N00014-16-1-2270. We acknowledge additional support from NSF Grants No. DMR-1609326 (S. M. G.) and No. DMR-1603243 (L. I. G.).

[1] Y. Nakamura, Y. A. Pashkin, and J.S. Tsai, Coherent Control of Macroscopic Quantum States in a SingleCooper-Pair Box, Nature (London) 398, 786 (1999).

[2] C. H. van der Wal, J. E. Mooij, T. P. Orlando, L. Levitov, L. Tian, and S. Lloyd, Josephson Persistent-Current Qubit, Science 285, 1036 (1999).

[3] D. Vion, A. Aassime, A. Cottet, P. Joyez, H. Pothier, C. Urbina, D. Esteve, and M. H. Devoret, Manipulating the Quantum State of an Electrical Circuit., Science 296, 886 (2002).
[4] H. Paik, D. I. Schuster, L. S. Bishop, G. Kirchmair, G. Catelani, A. P. Sears, B. R. Johnson, M. J. Reagor, L. Frunzio, L. I. Glazman et al., Observation of High Coherence in Josephson Junction Qubits Measured in a Three-Dimensional Circuit QED Architecture, Phys. Rev. Lett. 107, 240501 (2011).

[5] R. Barends, J. Kelly, A. Megrant, D. Sank, E. Jeffrey, Y. Chen, Y. Yin, B. Chiaro, J. Mutus, C. Neill et al., Coherent Josephson Qubit Suitable for Scalable Quantum Integrated Circuits, Phys. Rev. Lett. 111, 080502 (2013).

[6] G. Yan, Y. Liu, J. J. Raftery, and A. A. Houck, Suppression of Photon Shot Noise Dephasing in a Tunable Coupling Superconducting Qubit, arXiv:1603.01224.

[7] V. E. Manucharyan, J. Koch, L. I. Glazman, and M. H. Devoret, Fluxonium: Single Cooper-Pair Circuit Free of Charge Offsets, Science 326, 113 (2009).

[8] N. A. Masluk, I. M. Pop, A. Kamal, Z. K. Minev, and M. H. Devoret, Microwave Characterization of Josephson Junction Arrays: Implementing a Low Loss Superinductance, Phys. Rev. Lett. 109, 137002 (2012).

[9] A. Izmalkov, M. Grajcar, E. Il'ichev, T. Wagner, H.-G. Meyer, A. Y. Smirnov, M. H. S. Amin, A. M. van den Brink, and A. M. Zagoskin, Evidence for Entangled States of Two Coupled Flux Qubits, Phys. Rev. Lett. 93, 037003 (2004).

[10] J. B. Majer, F. G. Paauw, A. C. J. Ter Haar, C. J. P. M. Harmans, and J. E. Mooij, Spectroscopy on Two Coupled Superconducting Flux Qubits, Phys. Rev. Lett. 94, 090501 (2005).

[11] J. Majer, J. M. Chow, J. M. Gambetta, J. Koch, B. R. Johnson, J. A. Schreier, L. Frunzio, D. I. Schuster, A. A. Houck, A. Wallraff et al., Coupling Superconducting Qubits via a Cavity Bus, Nature (London) 449, 443 (2007).

[12] F. Yoshihara, Y. Nakamura, and J. S. Tsai, Correlated Flux Noise and Decoherence in Two Inductively Coupled Flux Qubits, Phys. Rev. B 81, 132502 (2010).

[13] Y. Chen, C. Neill, P. Roushan, N. Leung, M. Fang, R. Barends, J. Kelly, B. Campbell, Z. Chen, B. Chiaro et al., Qubit Architecture with High Coherence and Fast Tunable Coupling, Phys. Rev. Lett. 113, 220502 (2014).

[14] H. Meier, R. T. Brierley, A. Kou, S. M. Girvin, and L. I. Glazman, Signatures of Quantum Phase Transitions in the Dynamic Response of Fluxonium Qubit Chains, Phys. Rev. B 92, 064516 (2015).

[15] S. Gustavsson, J. Bylander, F. Yan, W. D. Oliver, F. Yoshihara, and Y. Nakamura, Noise Correlations in a Flux Qubit with Tunable Tunnel Coupling, Phys. Rev. B 84, 014525 (2011).

[16] M. T. Bell, J. Paramanandam, L. B. Ioffe, and M. E. Gershenson, Protected Josephson Rhombus Chains, Phys. Rev. Lett. 112, 167001 (2014).

[17] K. A. Matveev, A. I. Larkin, and L. I. Glazman, Persistent Current in Superconducting Nanorings, Phys. Rev. Lett. 89, 096802 (2002).

[18] We expect the resonant frequency of the array mode to be about $12.5 \mathrm{GHz}$, which is above the transition frequencies of interest for the molecule. We can then treat the array of junctions simply as a large inductance.

[19] F. Lecocq, I. M. Pop, Z. Peng, I. Matei, T. Crozes, T. Fournier, C. Naud, W. Guichard, and O. Buisson, Junction 
Fabrication by Shadow Evaporation without a Suspended Bridge, Nanotechnology 22, 315302 (2011).

[20] K. L. Geerlings, Improving Coherence of Superconducting Qubits and Resonators (Yale University Press, New Haven, CT, 2013).

[21] A. Blais, R. S. Huang, A. Wallraff, S. M. Girvin, and R. J. Schoelkopf, Cavity Quantum Electrodynamics for Superconducting Electrical Circuits: An Architecture for Quantum Computation, Phys. Rev. A 69, 062320 (2004).

[22] W. C. Smith, A. Kou, U. Vool, I. M. Pop, L. Frunzio, R. J. Schoelkopf, and M. H. Devoret, Quantization of Inductively-Shunted Superconducting Circuits, Phys. Rev. B 94, 144507 (2016).

[23] See Supplemental Material at http://link.aps.org/ supplemental/10.1103/PhysRevX.7.031037 for an extended discussion on decoherence and relaxation mechanisms. The supplemental material also shows data for manipulating the ground-state manifold using higher-energy excited states.

[24] F. Yoshihara, K. Harrabi, A. O. Niskanen, Y. Nakamura, and J.S. Tsai, Decoherence of Flux Qubits Due to $1 / f$ Flux Noise, Phys. Rev. Lett. 97, 167001 (2006).

[25] J. Bylander, S. Gustavsson, F. Yan, F. Yoshihara, K. Harrabi, G. Fitch, D. G. Cory, Y. Nakamura, J.-S. Tsai, and W. D. Oliver, Dynamical Decoupling and Noise Spectroscopy with a Superconducting Flux Qubit-Supplement, Nat. Phys. 7, 565 (2011).
[26] R. H. Koch, D. P. Divincenzo, and J. Clarke, Model for 1/f Flux Noise in SQUIDs and Qubits, Phys. Rev. Lett. 98, 267003 (2007).

[27] L. Faoro and L. B. Ioffe, Microscopic Origin of LowFrequency Flux Noise in Josephson Circuits, Phys. Rev. Lett. 100, 227005 (2008).

[28] P. Kumar, S. Sendelbach, M. A. Beck, J. W. Freeland, Z. Wang, H. Wang, C. C. Yu, R. Q. Wu, D. P. Pappas, and R. McDermott, Origin and Reduction of 1/f Magnetic Flux Noise in Superconducting Devices, Phys. Rev. Applied 6, 041001 (2016).

[29] L. B. Ioffe, M. V. Feigel'man, A. Ioselevich, D. Ivanov, M. Troyer, and G. Blatter, Topologically Protected Quantum Bits Using Josephson Junction Arrays, Nature (London) 415, 503 (2002).

[30] P. Brooks, A. Kitaev, and J. Preskill, Protected Gates for Superconducting Qubits, Phys. Rev. A 87, 052306 (2013).

[31] S. Gladchenko, D. Olaya, E. Dupont-Ferrier, B. Doucot, L. B. Ioffe, and M.E. Gershenson, Superconducting Nanocircuits for Topologically Protected Qubits, Nat. Phys. 5, 48 (2009).

[32] J. Cohen, W. C. Smith, M. H. Devoret, and M. Mirrahimi, Degeneracy-Preserving Quantum Non-demolition Measurement of Parity-Type Observables for Cat-Qubits, arXiv: 1611.01219. 